\title{
Heart Surface Motion Estimation Framework for Robotic Surgery Employing Meshless Methods
}

\author{
Evgeniya Bogatyrenko, Uwe D. Hanebeck, and Gábor Szabó
}

\begin{abstract}
A novel heart surface motion estimation framework for a robotic surgery on a stabilized beating heart is proposed. It includes an approach for the reconstruction and prediction of heart surface motion based on a novel physical model of the intervention area described by a distributedparameter system. Instead of conventional element methods, a meshless method is used for a spatial and temporal decomposition of this system. This leads to a finite-dimensional state-space form. Furthermore, the state of the resulting lumped-parameter system, which provides an approximation of the deflection and velocity of the heart surface, is dynamically estimated under consideration of uncertainties both occurring in the system and arising from noisy camera measurements. By using the estimation results, an accurate reconstruction of heart surface motion for the synchronisation of the surgical instruments is also achieved at occluded or non-measurement points.
\end{abstract}

\section{INTRODUCTION}

A recent development, so called off-pump surgery, can decrease the need for blood transfusion and morbidity of a on-pump coronary artery bypass surgery such as stroke or renal failure. With a particular area of the heart stabilized, the surgeon can operate on a beating heart without use of a heart-lung machine. However, some residual motion in the stabilized area remains [1], demanding the concentration of a surgeon on the heart motion during operation.

Some proposals have been made for a robotic surgery system with the goal to assist a surgeon by operating on a beating heart [2], [3]. This system consists of a measurement unit, such as a camera, an instrument robot, that moves surgical instruments according to the heart motion, control and image stabilization units. It will take over the challenges of the operation on a beating heart, such as the simultaneous vision and action coordination, and gives a surgeon the ability to dedicate his attention to the more complex tasks.

The main requirements on the robotic system for beating heart surgery are high accuracy, adaptation to any patient, and real-time operability. Hence, accurate measurements and a method for motion estimation and prediction for each point of interest on the heart surface are necessary. On the other hand, this is in opposition with low computational complexity and high speed.

For the estimation of heart motion, various approaches can be found in the literature. In general, they can be classified into methods based on the modeling of the heart as a physical

E. Bogatyrenko and U. D. Hanebeck are with Intelligent SensorActuator-Systems Laboratory, Institute for Anthropomatics, Universität Karlsruhe (TH), Germany. bogatyrenko@ira.uka.de, uwe.hanebeck@ieee.org

Gábor Szabó is with Heidelberg University Hospital, Germany Gabor.Szabo@urz.uni-heidelberg.de

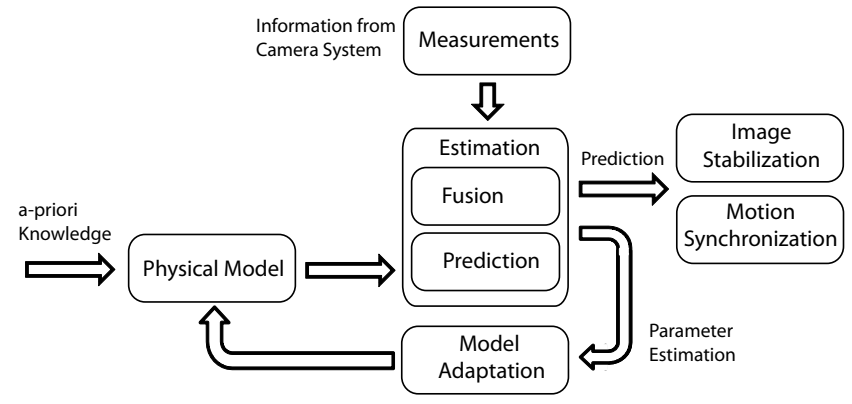

Fig. 1. Schematic overview of the robotic surgery system for motion and image synchronization during operations on a beating heart.

elastic body, and methods using other models. One of the methods from the second group [4] is based on the extraction of the respiration motion from the heartbeat motion using electrocardiogram and lung airflow information. A further approach of this group is described in [2], which applies the motion model of the heart's natural landmarks in order to reconstruct the motion of the heart surface. The first group dealing with physical elastic body models includes the approach [5], [6], where a model of the heart is used for the estimation of heart motion, and [7], [8], where a twodimensional model of the heart is proposed for magnetic resonance (MR) image analyses of cardiac kinematics.

The significant difference between the two groups of methods is that the ones based on the modeling of the heart as elastic body can better reconstruct motion between measurement points. The reason for this is that, by solving partial differential equations (PDE) characterizing the system, more accurate results can be achieved. This is because the physical characteristics of the system are used. It is also possible to reconstruct the heart motion at non-measurement points for a long time and thus, to bridge long disturbances during measurements when a sufficient physical model is provided. Therefore, the number of sensors and their measurement rates can be decreased. Also the interactions of the heart with the medical instruments can be considered more precisely. However, it should be pointed out that, unlike the other methods, the model-based estimation of a system, as complex as the heart, is computationally expensive and hardly possible in real-time. Not only does the simulation using models of complex geometry demand a lot of computational time, but also the numerical solution of the PDE with solvers, such as the finite-element (FEM), finite-volume (FVM) or finite-difference (FDM) methods, generates system-related response delays. 
In this paper, a novel framework for estimation of the heart surface motion for a robotic surgery system is presented. The main contribution is the prediction and reconstruction of the heart surface motion based on a new physical model described by a distributed-parameter system whose spatial and temporal decomposition is carried out by meshless methods. The purpose of the suggested physical model is not only to make a realistic reconstruction of heart motion but also to reduce computation time by simplifying the complex geometry of the heart.

In the next section, the problem of reconstructing the heart motion is described. The physical model of the heart as a thin plate is proposed in Section III. In Section IV, a short review of numerical solvers of PDEs is given in a context of robotic surgery systems. Section V discusses the spatial and temporal decomposition of the PDE. In Section VI, the proposed model of the thin elastic plate is evaluated in comparison with the analytic solution. Finally, estimation of the motion of non-measurement points is evaluated in an experiment on a pressure-regulated artificial heart (heart phantom). The discussion of the framework for model-based estimation closes with conclusions and an outlook on future work.

\section{PROBLEM FORMULATION}

The part of the robotic surgery system considered here is shown in Fig. 1. The fusion of measured data monitored by a camera system during heart operation and a physical model of the heart surface provided by the incorporation of a-priori information leads to the reconstruction of the distributed system of heart motion. The reconstructed information is required for motion synchronization and image stabilization. The estimation problem can be solved with filtering approaches, surveyed in [9]. For linear cases, the Kalman filter [10] and its derivatives can be used. In nonlinear cases, nonlinear estimation procedures, e.g the particle filter [11], the Gaussian Sum filter [12], should be applied. All these estimators require a system description in lumped-parameter state-space form.

At first, the motion of the heart surface should be characterized as a distributed phenomenon by means of a PDE. This formulation has to consider the physical characteristics as well as specific material properties and external forces that affect and deform the surface. Then, the distributedparameter system should be converted into lumped-parameter form, which can be achieved by numerical methods for solving PDEs. This dynamic lumped-parameter system model has to be transferred into the state-space form for estimating the state of a distributed system, such as heart surface motion in a robotic surgery system.

In order to meet the high demand for accuracy of the measurement system for robotic surgery, measurements must be taken on discrete points of the heart surface, as shown in Fig. 2. These markers can be randomly distributed on the heart surface. However, their systematic placement near the intervention area leads to a higher accuracy of the proposed framework and thus to a better feasibility, higher safety, and

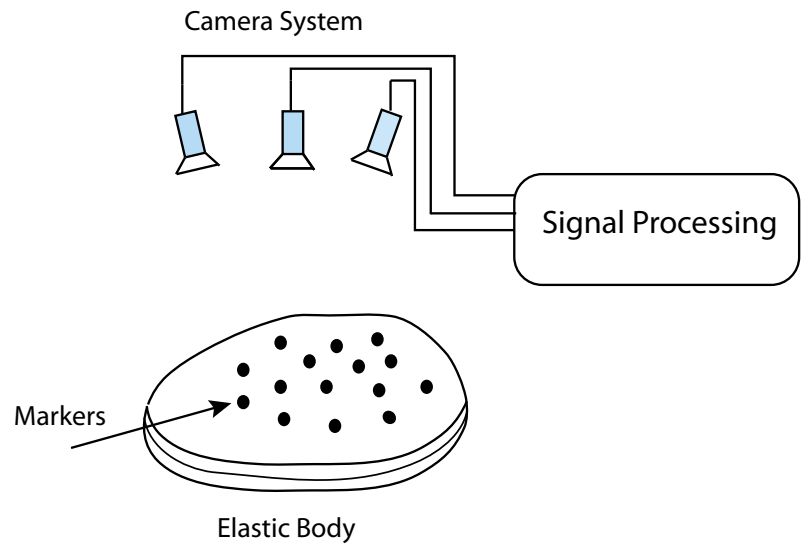

Fig. 2. Measurement system for accurate detection of heart motion.

more precise surgical interventions. The measured positions of these points can be used as a-priori information for the construction of the model, in order to avoid the matching or additional registration of the model to the heart surface.

\section{MODEL OF HEART SURFACE}

In this section, the behavior of the heart surface as a distributed phenomenon is formulated by means of a PDE. For this purpose, after a brief analysis of the heart geometry, the possible assumptions are justified and the thin plate model of the heart surface is proposed.

The purpose of the heart surface modeling is to simplify the complexity of the real system without serious loss in behavior, so that all off-pump bypass surgery operations can be perfomed with this approximation.

The geometry of the heart differs from patient to patient. For that reason, some medical statistical studies were undertaken in order to define the average heart wall dimensions, calculated under consideration of different patients. In [13], the average wall thickness of the left atrium is determined as about $2.5 \mathrm{~mm}$. In [14], [15], the average left ventricle wall is given as about $10 \mathrm{~mm}$. According to these studies, the thickness of the heart walls of the left ventricle as well as the left atrium is much smaller than their other two dimensions, such as length or width.

Under consideration of the heart surface geometry, the following assumptions are made to simplify the model of the heart surface in the intervention area. The shape of this area and boundary conditions are determined by a mechanical stabilizer, attached to the heart for the limitation and stabilization of the heart motion. This part of the heart surface is assumed to be of square shape with simply supported boundary conditions. The remaining out-of-plane deflections of the heart surface [1] are less than the heart wall thickness. Thus, the geometry of the heart and stabilization during operation allow to model the heart surface as a thin plate.

The classical deflection behavior of thin plates assumes that normals to the neutral surface of the undeformed plate remain straight and normal to the neutral surface during 


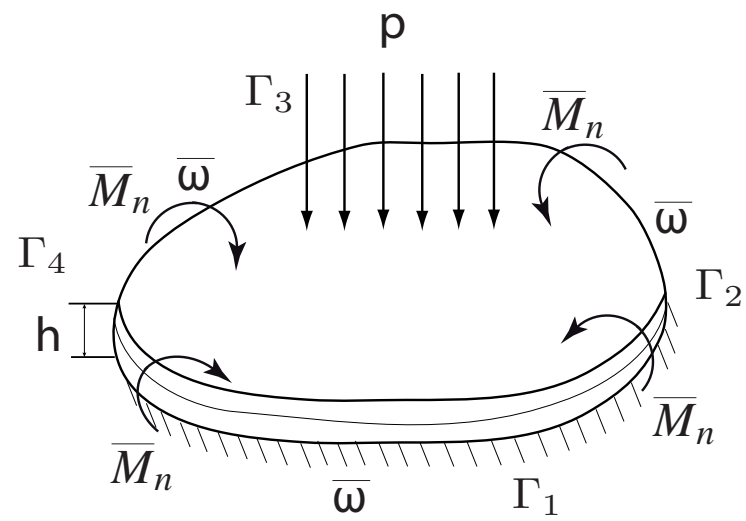

Fig. 3. Description of boundary conditions for the model, where on the boundaries $\Gamma_{1}, \Gamma_{2}, \Gamma_{3}, \Gamma_{4}$ the bending moments $\bar{M}_{n}$ and displacements $\bar{\omega}$ are imposed.

deformation or bending. Due to this so-called Kirchhoff assumption, all three displacement components are expressed in terms of the deflection in out-of-plane direction.

Because of small deformations due to the heart surface stabilization and restriction of operation area, the assumption that the thickness of the plate remains unchanged during the plate deformation, is made. For the same reasons, the heart surface can be treated as linear elastic, homogeneous, and isotropic. The transverse normal stresses were assumed to be negligible compared to the remaining non-zero stresses. The force affecting the heart surface is considered as transverse.

Thanks to these assumptions, the complexity of the heart surface model is decreased, and hence, a three-dimensional problem reduces to one involving only two dimensions.

According to the classical Kirchhoff thin plate theory, the static behavior of an homogeneous, isotropic, thin plate under bending is given by the Lagrange equation [16]. For the dynamic case the PDE describing the deflection of the heart surface by means of thin plate, shown in Fig. 3, can be written in the form

$$
\begin{gathered}
\nabla^{4} \omega(x, y, t)+\rho h \ddot{\omega}(x, y, t)=\frac{p(x, y, t)}{D}, \\
(x, y) \in \Omega_{1}=(0,1)^{2}, \quad t \geq 0,
\end{gathered}
$$

where $\omega$ denotes the deflection of the middle layer of the heart surface in z-direction, $\rho$ is the material density of the heart, $\nabla$ is the Nabla operator, $p$ is the transverse load, $h$ is the thickness of it and

$$
D=\frac{E h^{3}}{12(1-v)^{2}}
$$

is the bending stiffness of the heart area, which depends on the Young's modulus $E$ and Poisson's ratio $v$.

Considering the solution in a normalized domain $\Omega=$ $[0,1]^{2}$, taking into account the mechanical stabilization of the operation area, the following boundary and initial conditions

$$
\begin{aligned}
& \bar{\omega}(x, y, t)=g_{D}=0,(x, y) \in \Gamma_{1} \cup \Gamma_{2} \cup \Gamma_{3} \cup \Gamma_{4}, t \geq 0, \\
& \bar{M}_{n}(x, y, t)=g_{N}=0,(x, y) \in \Gamma_{1} \cup \Gamma_{2} \cup \Gamma_{3} \cup \Gamma_{4}, t \geq 0
\end{aligned}
$$

have to be imposed. Here, $g_{D}$ is referred to as a Dirichlet boundary condition and $g_{N}$, specifying a condition on the derivative, is the Neumann boundary condition. $\bar{M}_{n}$ are bending moments of a section of the plate perpendicular to the direction of the normal $n$ to the boundary. The boundaries of the plate are defined as

$$
\begin{aligned}
& \Gamma_{1}=\{(x, y): 0 \leq x \leq 1, y=0\}, \\
& \Gamma_{2}=\{(x, y): x=1,0 \leq y \leq 1\}, \\
& \Gamma_{3}=\{(x, y): 0 \leq x \leq 1, y=1\}, \\
& \Gamma_{4}=\{(x, y): x=0,0 \leq y \leq 1\} .
\end{aligned}
$$

and shown in Fig. 3. The applied bending moments are given by

$$
\begin{aligned}
\bar{M}_{n}=-D & \left\{v \nabla^{2} \omega+(1-v) .\right. \\
& \left.\left(\cos ^{2} \alpha \frac{\partial^{2} \omega}{\partial^{2} x}+\sin ^{2} \alpha \frac{\partial^{2} \omega}{\partial^{2} y}+\sin 2 \alpha \frac{\partial^{2} \omega}{\partial x \partial y}\right)\right\},
\end{aligned}
$$

where $\alpha$ is the angle between the plate surface and the normal of the boundary.

\section{MESHLESS METHODS FOR PARTIAL DIFFERENTIAL EQUATIONS}

This section deals with the analysis of different meshless methods regarding the application for model-based estimation in a robotic surgery system. After a brief description of the differences between meshless methods and classical methods for solving PDEs, their advantages and drawbacks are discussed.

\section{A. Overview of methods}

A variety of meshless methods was considered in [17], [18], [19], [20]. The obvious advantage of these methods in comparison to traditional numerical methods, such as FEM, FDM, or FVM, is that no information on the relationship between the nodes is required and thus no element mesh is needed. Since meshless discretization techniques are only based on a set of points, these methods are better suited to handle changes in geometry of the domain of interest [20]. Thus, by application of meshless methods in heart modeling, the changes of the heart surface, caused by surgical interventions, can be traced more precisely. Another advantage is easier adaptive analysis. In particular, when the mesh becomes extremely skewed or compressed because of large deformations or discontinuities, an adaptive remeshing or a node refinement must be performed in order to maintain reasonable numerical accuracy. In conventional methods, the enrichment is difficult due to the need for element connectivity. In meshless methods it can be achieved by adding or removing points.

Without the need for a predefined mesh, the meshless methods result in higher computational efficiency and better accuracy by handling of large deformations or discontinuities. Concerning the robotic surgery system, they can 
contribute to easier adaptation of the model to any patient, give flexibility in handling surgical interventions, and reduce the time for initialization of the robotic surgery system.

\section{B. Classification of meshless methods}

While the major difference between meshless methods comes from the techniques for interpolating trial and test functions, only general groups of them will be taken into account here. According to computational modeling, the meshless methods may be categorized into two different classes: those, where the use of background cells is necessary for the integration, and those, where no mesh is required at all. A method in a first class is Smoothed Particle Hydrodynamics (SPH) [21], [22]. Here the predefinion of particles for their volumes or masses still requires some kind of cells. Furthermore, the Element-Free Galerkin Method (EFG) [23], based on the global Galerkin Weak Formulation, needs background cells for the integration of system matrices. The second class contains the following meshless methods: the Meshless Local Petrov-Galerkin Method (MLPG) [24], the Spectral Method (SM) [25], and the Collocation Method (CM) [26]. Without need for cells the latter methods are better suited for adaptation and enrichment. They will now be analyzed regarding to their application for robotic surgery system.

1) Meshless Local Petrov-Galerkin Method: The MLPG method is based on the definition of simple subdomains, thus, avoiding the background cells. Contrary to the EFG, where the trial and test functions are chosen from the same function space, in MLPG the nodal trial and test functions can be different. This makes the MLPG method very flexible. Hence, when the Dirac delta distribution is used as the test function, the local $\mathrm{CM}$ without numerical integration results. This is the simplest form of the MLPG. Setting the Heaviside function as test function leads to an integration over regular boundaries in order to construct the stiffness matrix. Despite higher complexity in comparison to the use of the Dirac delta distribution as test function, a better accuracy and stability can be achieved. The drawback of using the Heaviside function is the higher amount of computations for solving of PDEs because of the non-symmetrical stiffness matrix and the need for integration.

2) Spectral Method: The SM is known as highly accurate solver for PDEs [20]. The fundamental principle of these methods is to use a set of very smooth and global basis functions, such as polynomials, to represent the approximate solution of the PDE through the evaluation of the derivative of this solution. Frequently, orthogonal polynomials, such as Chebyshev polynomials, are used as basis functions along with the corresponding Chebyshev points. The special arrangement of grid points is defined through minimal-energy configurations associated with the inverse linear repulsion between these points. According to [25], the effect of using these clustered points on the accuracy of the interpolation is dramatic. Thus, the main drawback of these methods for the described robotic surgery system is the use of a special arrangement of grid points for achieving high precision.
Because there is only access to accurate measurements on some points of the heart surface, as shown in Fig. 2, the cluster of points cannot be chosen freely.

3) Collocation Method: The CM makes the approximation of the dependent variables by suitable polynomials or functions according to their values in the collocation points, corresponding to a suitable discretization of the space variable. Supposing that the error vanishes at certain collocation points, this method has three major advantages. Since no integration is required, the construction of the final system of equations is efficient. Moreover, the shape functions are only evaluated at nodes rather than at integration points as in other methods. Due to the fact that only the points are considered, the refinement and adaptive analysis can be realized very fast. Furthermore, this method efficiently deals with nonlinearities [27]. However, the precision and existence of a solution strongly depends on the distribution and amount of collocation points.

\section{Summary}

All in all, the three methods MLPG, SM, CM can be seen as suitable for modeling of the heart surface, regarding the model-based estimation in robotic surgery system. In this paper, we focus on the modeling of the heart surface as the thin plate with CM. Other methods, such as MLPG or SM, will be evaluated in further research.

Finally, it should be noted, that SPH and CM are often referred to as strong form solutions, while EFG and MLPG are based on the weak form solutions. Despite the fact that the discretized equation systems based on the weak form are more stable and can give much more accurate results [17], the methods referred to as strong form solutions are very suitable for real-time applications because of low computational complexity. For all methods, the selection of the best number of nodes is of paramount importance for solving time-dependent problems, as increasing the number of nodes reduces the interpolation error but increases the error related to time integration.

\section{SOLUTION OF PARTIAL DIFFERENTIAL EQUATIONS}

This section covers the conversion of the PDEs (1) and (2) into a set of ordinary differential equations (ODE). For this purpose, the spatial and temporal decomposition is carried out by solving of PDEs with a non-symmetric collocation method.

This method is described in [26], where the author comments on its superior performance in terms of computational complexity and accuracy when compared to finite difference methods.

The basic idea of collocation methods is to use a set of very smooth and global basis functions $\phi_{j}(p), j=1, \ldots, N$, such as polynomials, to approximate the solution of the PDE. Thus, the plate deflection in z-direction $\omega^{i}$ at collocation point $i$ is approximated via

$$
\omega^{i}(p, t)=\sum_{j=1}^{N} c_{j}(t) \phi_{j}(p), \quad p \in \mathbb{R},
$$


where $N$ is the total number of collocation points and $c_{j}$ are the weighting coefficients to be determined. The basis functions, depending on space, are infinitely smooth and are given as

$$
\phi_{j}(p)=\varphi\left(\left\|p_{i}-\xi_{j}\right\|\right)=\varphi(r), i, j=1, \ldots N,
$$

where $\varphi(r)$ is positive-definite univariate basic function, $\Xi=\left\{\xi_{1}, \ldots, \xi_{N}\right\}$ presents its center points, and $P=$ $\left\{p_{1}, \ldots, p_{N}\right\} \subset \Omega$ are collocation points. In the following, inverse multiquadrics

$$
\varphi(r)=\frac{1}{\sqrt{1+(e \cdot r)^{2}}}
$$

are used with the scaling parameter $e>0$ as positive-definite basis function for interpolation. A small value of $e$ produces flat basis functions, whereas a large value of $e$ leads to very steep functions. The accuracy of the fit will improve with decreasing $e$, while the stability will decrease, resulting in less reliable numerical results.

Substituting the finite expansion (4), containing the spatial and temporal decomposition of the PDE solution, the system of PDEs (1) and (2) is converted to the ODE system.

\section{State-Space Form}

This section is devoted to the derivation of the discretetime system and measurement equations for the purpose of estimation of the heart surface motion.

\section{A. System Equation}

The unconditionally and absolutely stable implicit Euler integration method is used for time-discretization of ODEs describing the dynamic behavior of the heart surface area, modeled by means of a thin plate. The discretization of the plate deflection in z-direction is carried out according to the standard backward difference approximation

$$
\frac{\partial \omega^{i}\left(x, y, t=t_{k+1}\right)}{\partial t} \approx \frac{\omega_{k+1}^{i}-\omega_{k}^{i}}{\Delta t},
$$

where $\Delta t$ is the sampling time, the superscript $i$ and the subscript $k$ in $\omega_{k}^{i}$ denote the value of the distributed system at collocation point $i$ and at time step $k$. The substitution of the approximation of the plate deflection in z-direction $\omega_{k+1}^{i}$ and $\omega_{k}^{i}$ via (4) into (1) and (2) leads to the following system equation

$$
\underline{x}_{k+1}=\mathbf{A}_{k} \underline{x}_{k}+\mathbf{B}_{k} \underline{u}_{k+1}+\underline{w}_{k}
$$

where the state vector

$$
\underline{x}_{k}=\left[\underline{c}_{k}, \underline{\beta}_{k}\right]^{T}
$$

is the vector of the unknown weighting coefficients $c_{j}$ and their time derivatives $\beta_{j}$ at time step $k$. The vector $\underline{w}_{k}$ represents the noise and modeling errors.

The matrices $\mathbf{A}_{k}$ and $\mathbf{B}_{k}$ and the input vector $\underline{u}_{k+1}$ are determined by the linear system equation

$$
\left[\begin{array}{l}
\mathbf{A}_{L} \\
\mathbf{A}_{u} \\
\mathbf{A}_{M_{n}}
\end{array}\right] \underline{x}_{k+1}=\left[\begin{array}{l}
\frac{f}{\bar{g}} \\
\underline{\bar{g}}_{N}
\end{array}\right]
$$

Furthermore, the construction of this system is considered for every partition of the state vector (7) at time step $k+1$. For that purpose, the set of collocation points in a domain $\Omega=[0,1]^{2}$ is split into a set $\Omega_{1}$ of interior collocation points and a set $\Gamma_{1} \cup \Gamma_{2} \cup \Gamma_{3} \cup \Gamma_{4}$ of boundary points.

For the partition of the state vector $\underline{c}_{k+1}$, corresponding to the out-of-plane deflection of heart surface, the matrices $\mathbf{A}_{u}$ and $\mathbf{A}_{M_{n}}$ describe the displacement of boundary nodes $p_{i} \in \Gamma_{1} \cup \Gamma_{2} \cup \Gamma_{3} \cup \Gamma_{4}$ and are defined as

$$
\begin{aligned}
& \left(\mathbf{A}_{u}\right)_{i j}=\left.[\varphi(\|\underline{p}-\underline{\xi}\|), 0]\right|_{\underline{p}=p_{i}, \underline{\xi}=\xi_{j},} \\
& \left(\mathbf{A}_{M_{n}}\right)_{i j}=\left.\left[B_{M_{n}} \varphi(\|\underline{p}-\underline{\xi}\|), 0\right]\right|_{\underline{p}=p_{i}, \underline{\xi}=\xi_{j}},
\end{aligned}
$$

where $\xi_{j} \in \Gamma_{1} \cup \Gamma_{2} \cup \Gamma_{3} \cup \Gamma_{4}$. Because of mechanical stabilization of the heart during operation, the operation area was assumed to have time-independent, simply supported boundary conditions. Therefore, the differential operator $B_{M_{n}}$ of bending moments (3) is given as

$$
\begin{aligned}
B_{M_{n}}=-D & \left\{v \nabla^{2}+(1-v) .\right. \\
& \left.\left(\cos ^{2} \alpha \frac{\partial^{2}}{\partial^{2} x}+\sin ^{2} \alpha \frac{\partial^{2}}{\partial^{2} y}+\sin 2 \alpha \frac{\partial^{2}}{\partial x \partial y}\right)\right\} .
\end{aligned}
$$

The matrix $\mathbf{A}_{L}$ determines the displacement of internal nodes $p_{i} \in \Omega_{1}=(0,1)^{2}$ and is generated as

$$
\left(\mathbf{A}_{L}\right)_{i j}=\left.[\varphi(\|\underline{p}-\underline{\xi}\|),-T \varphi(\|\underline{p}-\underline{\xi}\|)]\right|_{\underline{p}=p_{i}, \underline{\xi}=\xi_{j}},
$$

where $\xi_{j} \in \Omega_{1}, T$ denotes the sampling time. The force vector contains the approximation of the deflection in $\mathrm{z}$ direction at the time step $k$

$$
(\underline{f})_{i}=\left.\varphi(\|\underline{p}-\underline{\xi}\|)\right|_{\underline{p}=p_{i}, \underline{\xi}=\xi_{j}} c_{j}\left(t=t_{k}\right) .
$$

For the second partition of the state vector $\beta_{k+1}$, corresponding to the velocity of the motion out-of-plane direction, the block of matrix $\mathbf{A}_{L}$ for internal nodes $p_{i} \in \Omega_{1}=(0,1)^{2}$ is generated as follows

$$
\left(\mathbf{A}_{L}\right)_{i j}=\left.[L \varphi(\|\underline{p}-\underline{\xi}\|), \varphi(\|\underline{p}-\underline{\xi}\|)]\right|_{\underline{p}=p_{i}, \underline{\xi}=\xi_{j}} .
$$

The differential operator $L$ depends on the sampling time $T$

$$
L=\frac{T \nabla^{4}}{\rho h}
$$

So does the force vector

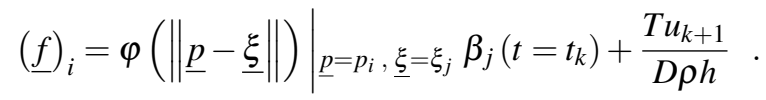

By solving the linear system (8) for vector coefficients $\underline{x}_{k+1}$, the deflection $\omega_{k+1}$ of the heart surface in z-direction and its velocity at time step $k+1$ can be determined by means of the approximation (4). 


\section{B. Measurement Equation}

In this section, the discrete-time measurement equation, based on the camera measurements, is derived.

Since the displacements of the markers are observed and used to improve the estimated state, distributing more markers on the heart surface leads to higher accuracy in the estimation.

A relation between the measurements of marker displacements $\underline{\underline{y}}=\left[\underline{y}_{z}, \underline{y}_{x}, \underline{y}_{y}\right]^{T}$ and the state vector $\underline{x}_{k}$ is given in the form

$$
\underline{\hat{y}}=\mathbf{H}_{k} \underline{x}_{k}+\underline{v},
$$

where $\mathbf{H}_{k}$ is the measurement gain matrix and $\underline{v}$ defines the measurement uncertainties. The matrix $\mathbf{H}_{k}$ for the measured displacement in z-direction $\underline{y}_{z}=\underline{\omega}$ at time step $k$ is determined by the equation

$$
\underline{\hat{y}}_{z}=\boldsymbol{\Phi}(\|\underline{p}-\underline{\xi}\|) \underline{c}_{k}^{T}
$$

where $\Phi$ is the matrix of radial basic functions (5).

The displacements parallel to the undeformed middle layer of the plate in the $\mathrm{x}$ - and $\mathrm{y}$-direction, $\underline{y}_{x}$ and $\underline{y}_{y}$, at a distance $z$ from the neutral surface can be expressed by

$$
\underline{y}_{x}=-z \frac{\partial \underline{\omega}}{\partial x}, \underline{y}_{y}=-z \frac{\partial \underline{\omega}}{\partial y} .
$$

It is clearly shown here that all three displacement components are expressed in terms of the deflection vector $\underline{\omega}$, due to the Kirchhoff assumption. From this it follows that the measurement gain matrix $\mathbf{H}_{k}$ for the computation of the displacements of the heart surface in the $\mathrm{x}$ - and $\mathrm{y}$-direction, approximated by

$$
\begin{aligned}
& \widehat{\underline{y}}_{x}=-z \frac{\partial \boldsymbol{\Phi}(\|\underline{p}-\underline{\xi}\|)}{\partial x} \underline{c}_{k}^{T}, \\
& \widehat{\hat{y}}_{y}=-z \frac{\partial \boldsymbol{\Phi}(\|\underline{p}-\underline{\xi}\|)}{\partial y} \underline{c}_{k}^{T}
\end{aligned}
$$

contains the spatial derivatives of the radial basic function.

Thus, the PDE (1), describing the distributed phenomenon, such as heart surface motion, was converted by employing the meshless collocation method from an infinitedimensional state-space form into a discrete-time lumpedparameter form. Then, under consideration of endogenous uncertainties, e.g., modeling errors, noise of control function $f$, and uncertainties by the definition of boundary conditions, this system was converted into the finite-dimentional statespace form, which is required for solving of estimation problems. Due to the fact that the system equation (6) and the measurement equation (10) are linear, it is sufficient to use the Kalman filter to obtain the best possible estimate for the system state characterizing the motion of heart surface.

\section{SIMULATION RESULTS}

In this section, the performance of the proposed modeling framework will be demonstrated by means of a simulation and an experiment.

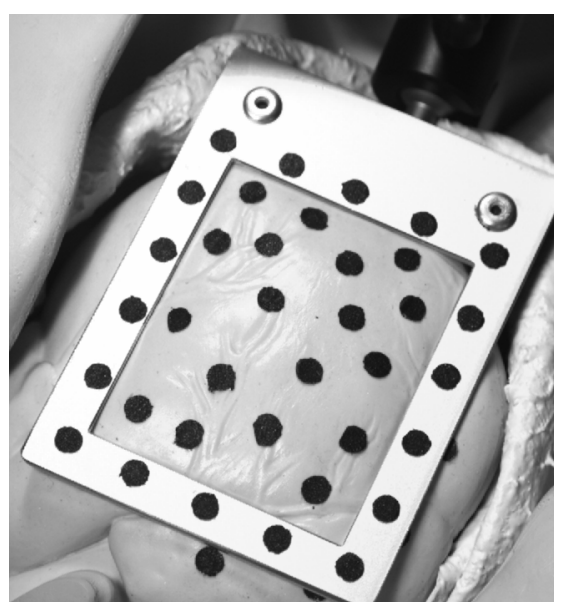

Fig. 4. Experimental setup for the evaluation of the proposed modeling framework.

First, the simulation results of a small heart area modeled by a thin plate are presented and compared with the exact solution of the thin plate's deformation. The purpose of this simulation is to evaluate the accuracy of the non-symmetric collocation meshless method employed for a conversion of the heart surface model into lumped-parameter form.

The collocation points are initialized by use of the coordinates of the discrete measurement points attached to the heart phantom, as shown in Fig. 4.

Because of the assumption that the operation area is mechanically stabilized, a square plate describing the heart area of size $a=b=10 \mathrm{~cm}$ is considered. The thickness of the plate $h=10 \mathrm{~mm}$ corresponds to the average value of the left ventricle wall thickness. During simulations, the elastic plate with Poisson's ratio $v=0.3$ and Young's modulus $E=$ $10920 \mathrm{Nm}^{-2}$ is subjected to a uniformly distributed transverse dynamic load $p=p_{0} \theta(t)$ with the frequency $1.17 \mathrm{~Hz}$ corresponding to the average heartbeat rate for adults. The plate under the peak load $p_{0}=10 \mathrm{~Pa}$ and its deflections in the z-direction are presented in Fig. 5. Because the frequency of the excitation is small in comparison to the natural circular frequency of the plate, the displacements correspond to those obtained from the static loading. The values of simulated deformation in the z-direction are compared to the deformations determined by the Navier solution for simply supported rectangular plates discussed in [16]

$$
\omega(x, y)=\frac{16 p_{0}}{\pi^{6} D} \sum_{m=1}^{\infty} \sum_{n=1}^{\infty} \frac{\sin \frac{m \pi x}{a} \sin \frac{n \pi y}{b}}{m n\left(\frac{m^{2}}{a^{2}}+\frac{n^{2}}{b^{2}}\right)^{2}},
$$

where $\mathrm{m}$ and $\mathrm{n}$ are odd integers, which influence the accuracy of the solution by determining the number of series terms. By setting $m=n=11$, a high accuracy is achieved in the analytical solution. As illustrated in Fig. 6, the maximum error occurs near the boundary. In the middle of the plate there is a displacement of about $4 \mathrm{~mm}$ with the absolute error of about $5 \mu \mathrm{m}$. The high accuracy of reconstruction in the middle of the plate is of significant importance because of the possibility to restrict this area by means of the mechanical 


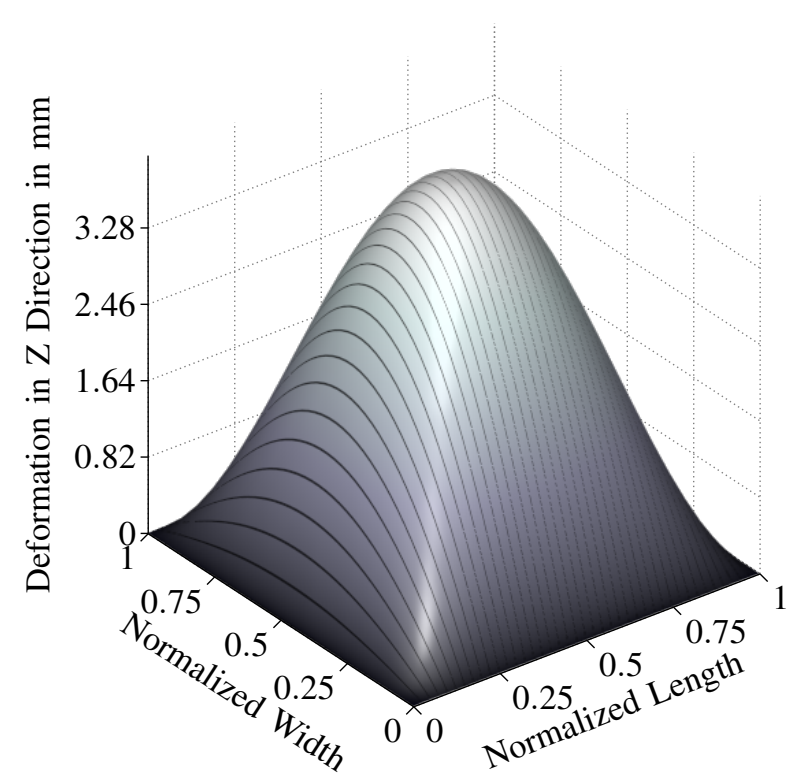

Fig. 5. Simulated deformation in z-direction of simply supported thin plate under transverse load.

stabilizer. The root mean square error of the maximum deflection is $8 \mu \mathrm{m}$.

Therefore, by the conversion of the heart surface model into lumped-parameter form with the non-symmetric collocation method, a high accuracy of reconstruction is achieved.

In the following experiment, the evaluation of the estimation is demonstrated. The goal is the reconstruction of heart surface motion at non-measurement points using both a physical model and measurements obtained by a camera system. It is important to note that the uncertainties arising from noisy measurements and occurring in the physical model are considered.

For the purpose of evaluation, the pressure-regulated heart phantom is controlled by a sinusoidal dynamic load with the frequency of $1.2 \mathrm{~Hz}$ and an amplitude of about $100 \mathrm{hPa}$. The setup of the experiment is shown in Fig. 4, where the stabilization device is attached to the heart surface. The parameters of the heart phantom were assumed to be timeinvariant and exactly known. The Kalman filter is used to obtain the state estimate for the linear system, characterizing the motion of the heart surface phantom. For z-direction, the estimated solution and its $3 \sigma$-bound at a non-measurement point is visualized in Fig. 7. The reconstruction of the heart surface motion at a point whose measurements were not considered by the estimation corresponds well to the measurements.

\section{CONCLUSIONS AND FUTURE WORK}

In this paper, a novel framework for the estimation of heart surface motion based on a physical model is proposed. The purpose is the synchronization of surgical instruments during operations on a mechanically stabilized beating heart.

Thanks to the assumptions based on the geometry of the heart and a mechanical stabilization of a beating heart during

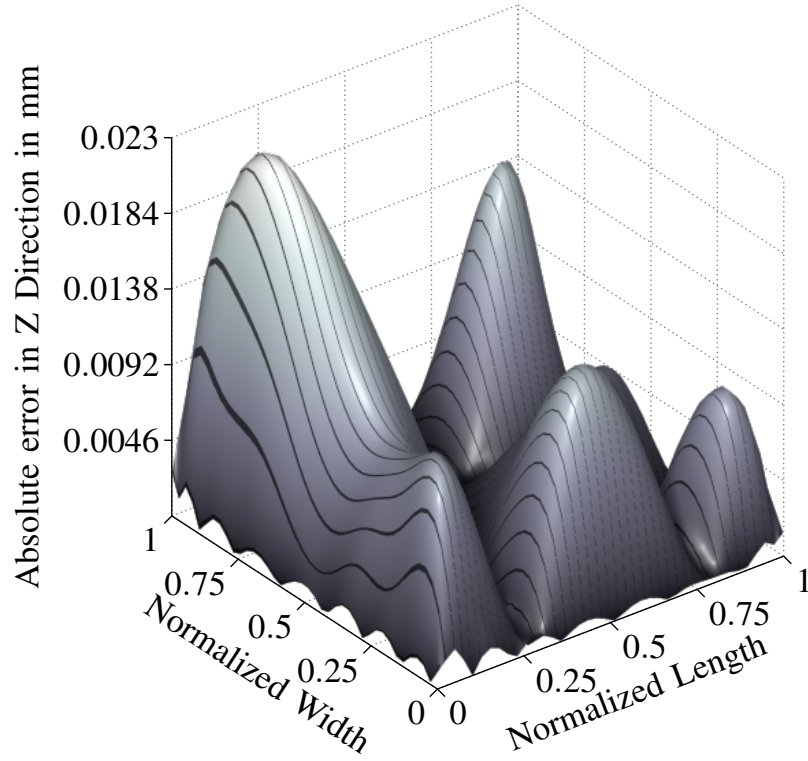

Fig. 6. Distribution of absolute error in z-direction compared to the exact solution for simply supported thin plate under transverse load.

operation the reduction of the three-dimensional modeling problem to a two-dimensional one is possible. This leads to a lower computational complexity of the heart surface model and thus, contributes to the real-time operability of the robotic surgery system.

Furthermore, new methods for the conversion of a distributed-parameter system, describing heart surface motion, to a lumped-parameter system required for modelbased estimation, were introduced. Applying these methods in robotic surgery applications provides novel prospects to efficient robotic surgery on the beating heart. Since meshless methods are only based on a set of points, they are well suited to handle changes in the geometry of the model. This allows precise tracing of the changes of the heart surface caused by surgical interventions. Thanks to remeshing or adaptation analysis without the need of connection information between the points, there is the possibility of a simple adaptation of the model for any patient and even on the complex geometries of the heart.

The performance of one of the proposed modeling approaches was demonstrated by comparing simulation results with the exact analytic solution and high accuracy was achieved. This framework was evaluated with model-based estimation by means of the Kalman filter in an experiment involving a pressure-regulated heart phantom. It should be noted, that for the high accuracy of the model, exact information about the control function, boundary conditions, and parameters of the distributed system is required. Thus, for the adaption of the model to any patient, the uncertainties of the force affecting the heart surface, modeling parameters, and approximations errors should be taken into account. Therefore, the derived model should be used with combined parameter and state estimation approaches in order to reconstruct the deflection of the heart surface on any point. 


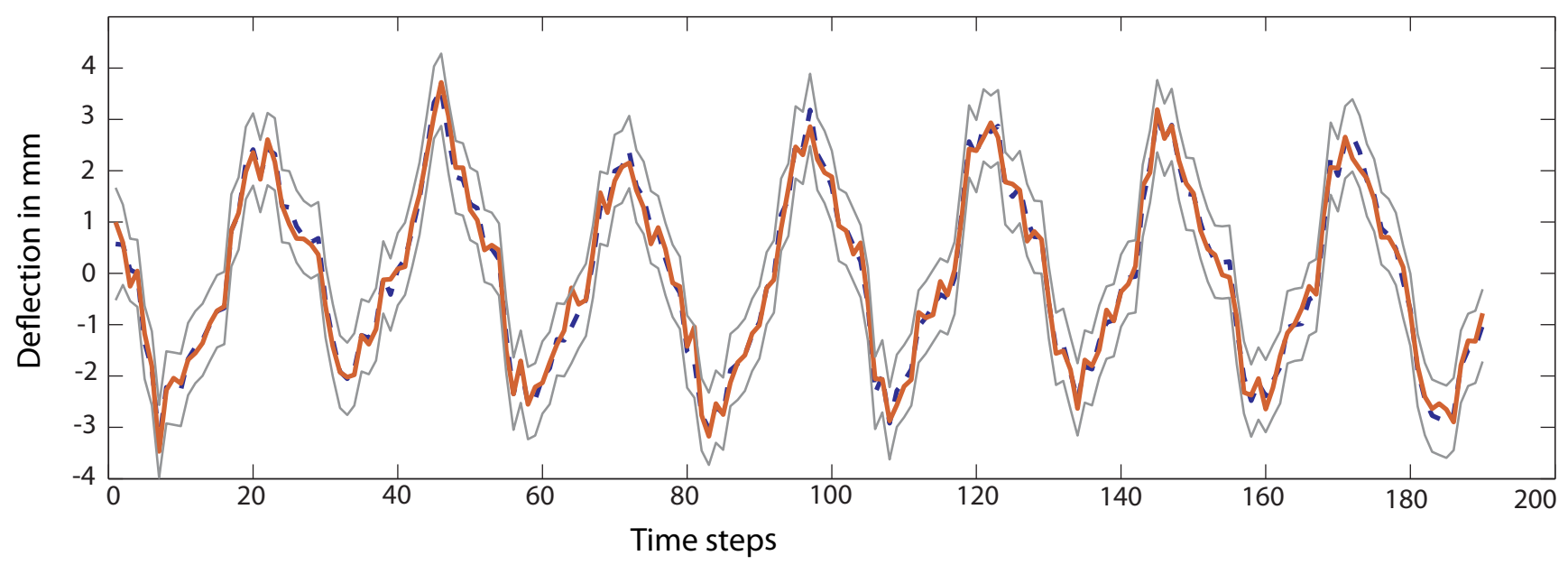

Fig. 7. Comparison of estimated solution (blue) and its $3 \sigma$-bounds (gray) with the corresponding measurements (red) for a point whose measurements were not considered by the estimation.

\section{ACKNOWLEDGEMENTS}

This work was partly supported by the German Research Foundation (DFG) within the Research Training Group RTG 1126 Intelligent Surgery.

\section{REFERENCES}

[1] P. Cattina, H. Daveb, J. Gruenenfelder, G. Szekelya, M. Turin, and G. Zuend, "Trajectory of Coronary Motion and its Significance in Robotic Motion Cancellation," European Journal of Cardio-thoracic Surgery, vol. 25, pp. 786-790, 2004.

[2] T. Ortmaier, M. Groeger, D. H. Boehm, V. Falk, and G. Hirzinger, "Motion Estimation in Beating Heart Surgery," IEEE Transactions on Biomedical Engineering, vol. 52, 2005.

[3] Y. Nakamura, K. Kishi, and H. Kawakami, "Heartbeat Synchronization for Robotic Cardiac Surgery," Proceedings of the IEEE international Conference on Robotics and Automation, Seoul, Korea, 2001.

[4] L. Cuvillon, J. Gangloff, M. de Mathelin, and A. Forgione, "Toward Robotized Beating Heart TECABG: Assessment of the Heart Dynamics Using High-Speed Vision," J. Duncan and G. Gerig (Eds.) (MICCAI 2005), LNCS 3750, p. 551558, 2005.

[5] K. Roberts and U. D. Hanebeck, "Motion Estimation and Reconstruction of a Heart Surface by Means of 2D-/3D-Membrane Models," Proceedings of 21st International Congress and Exhibition on Computer Assisted Radiology and Surgery (CARS 2007), Berlin, pp. 243-245, 2007.

[6] T. Bader, A. Wiedemann, K. Roberts, and U. D. Hanebeck, "Modelbased Motion Estimation of Elastic Surfaces for Minimally Invasive Cardiac Surgery,", IEEE International Conference on Robotics and Automation ( ICRA 2007), Roma, Italy, 2007.

[7] P. Shi and H. Liu, "Stochastic Finite Element Framework for Cardiac Kinematics Function and Material Property Analysis," T. Dohi and R. Kikinis (Eds.) (MICCAI 2002), LNCS 2488, pp. 634-641, 2002.

[8] H. Liu and P. Shi, "Meshfree Representation and Computation: Applications to Cardiac Motion Analysis," C.J. Taylor and J.A. Noble (Eds.) (IPMI 2003), LNCS 2732, pp. 560-572, 2003.

[9] D. Simon, Optimal State Estimation. Hoboken, Wiley-Interscience, 2006.

[10] R. E. Kalman, "A New Approach to Linear Filtering and Prediction Problems," Transactions of the ASME Journal of Basic Engineering, vol. 82, pp. 35-45, 1960.

[11] A. Doucet, N. d. Freitas, and N. E. Gordon, Sequential Monte Carlo Methods in Practice. Springer-Verlag, 2001.

[12] D. Alspach and H. Sorenson, "Nonlinear Bayesian estimation using Gaussian sum approximations," Automatic Control, IEEE Transactions, vol. 17 (4), 1972.
[13] P. Platonov, V. Ivanov, S. Y. Ho, and L. Mitrofanova, "Left Atrial Posterior Wall Thickness in Patients with and without Atrial Fibrillation: Data from 298 Consecutive Autopsies," Journal of Cardiovascular Electrophysiology, vol. 19, pp. 689-692, 2008.

[14] M. Sehgal, K. Hirose, J. E. Reed, and J. A. Rumberger, "Regional Left Ventricular Wall Thickness and Systolic Function during the First Year After Index Anterior Wall Myocardial Infarction: Serial Effects of Ventricular Remodeling," International Journal of Cardiology, vol. 53, pp. 45-54, 1996.

[15] K. L. Dixon, A. Fung, and A. Celler, "The Effect of Patient, Acquisition and Reconstruction Variables on Myocardial Wall Thickness as Measured from Myocardial Perfusion SPECT Studies." IEEE Nuclear Science Symposium Conference Record, 2004.

[16] S. Timoshenko and S. Woinowsky-Krieger, Theory of Plates and Shells. McGraw-Hill, Inc., 1959.

[17] G.-R. Liu, Mesh Free Methods: Moving Beyond the Finite Element Methods. CRC Press, 2003.

[18] S. N. Atluri, Methods of Computing Modelling in Engineering and the Science. Volume 1. Tech Science Press, 2005.

[19] T. Rabczuk, S. Bordas, M. Duflot, and N. V. Phu, "Meshless Methods: a Review and Computer Implementation Aspects," Mathematics and Computers in Simulation (IMACS 2008), vol. 79, pp. 763-813, 2008.

[20] G. E. Fasshauer, Meshfree Approximation Methods with Matlab. Interdisciplinary Mathematical Sciences - Vol. 6, World Scientific Publishing, 2007.

[21] R. A. Gingold and J. J. Monaghan, "Smoothed Particle Hydrodynamics: Theory and Application to non-spherical stars," Monthly Notices Roy. Astronom. Soc., vol. 181, pp. 375-389, 1977.

[22] L. B. Lucy, "A Numerical Approach to the Testing of the Fission Hypothesis," The Astronomical Journal, vol. 82, pp. 1013-1024, 1977.

[23] T. Belytschko, Y. Y. Lu, and L. Gu, "Element-free Galerkin methods," International Journal for Numerical Methods in Engineering, vol. 37, pp. 229-256, 1994.

[24] S. N. Atluri and S. Shen, "The Meshless Local Petrov-Galerkin (MLPG) Method: A Simple, Less-costly Alternative to the Finite Element and Boundary Element Methods," Tech Science Press, CMES, vol. 3 (1), pp. pp.11-51, 2002.

[25] L. N. Trefethen, Spectral Methods in Matlab. SIAM, Philadelphia, 2000.

[26] E. Kansa, "Multiquadratic - a Scattered Data Approximation Scheme with Applications to Computational Fluid Dynamics. Solution to Parabolic, Hyperbolic and Elliptical Partial Differential Equations," Computer and Mathematics with Applications, vol. 19 (8-9), pp. 147$161,1990$.

[27] N. Bellomo, B. Lods, R. Revelli, and L. Ridolfi, Generalized Collocation Methods. Solutions to Nonlinear Problems. Birkhaeuser Boston, 2008. 